\title{
GENOTYPE BY YIELD*TRAIT COMBINATION BIPLOT APPROACH TO EVALUATE SESAME GENOTYPES ON MULTIPLE TRAITS BASIS
}

\author{
Seyni BOUREIMA*, Abdoua YAOU \\ University Dan Dicko Dankoulodo of Maradi, Faculty of Agronomy and Environmental Sciences, \\ Department of Sciences and Crop Production, Maradi, NIGER \\ Corresponding author: b.seyni@gmail.com
}

Received: 01.11.2019

\begin{abstract}
The objectives of this study were to understand the relationships among various traits of the crop and to assess genotype profile suitable for semi-arid rainfed conditions. Experiments were conducted over 2 years at five locations to evaluate the performance of 10 sesame varieties in Randomized Complete Block Design. Significant variations were observed among the varieties for most studied traits with a consistent Genotype $X$ Environment interaction. The genotype by yield*trait combination (GYT) biplot which captured $94.73 \%$ of the total variation showed that the key traits for a superior sesame variety in rainfed conditions of semi-arid regions are unbranched or few branches, earliness, medium height and short HIPC. The GYT biplot identified some superior sesame varieties as good for a specific trait or a group of traits. Based on the levels in combining yield with other target traits and genotype trait profiles, variety DS 01 was ranked first followed by SN 403. The GYT biplot approach used in this study was found to be very helpful in identifying key traits for superior genotypes of sesame in semi-arid conditions of Niger.
\end{abstract}

Keywords: Biplot, multiple traits, selection, Sesamum indicum L.

\section{INTRODUCTION}

Elite varieties must be identified on multiple traits basis through multi-environment trials (Yan and Rajcan, 2002) and must be top performers for a number of target traits to assure that the selected genotypes perform well in a set of environments and meet the demand from the producers, consumers and processors (Yan and FrégeauReid, 2018). Identifying superior individuals out of a population of genotypes is a key part of plant breeding process. Two important hindrances are the genotype-byenvironment interaction (GE) on key traits and the unfavorable associations among desired traits. Although yield remains the primary target trait for most breeding programs, in practice breeders generally select considering several traits (Bos and Caligari, 2008). However, breeding for two or more traits is generally more difficult than breeding for a single trait alone (Bernardo, 2010). The difficulties arise when two traits are unfavorably associated, selection for the first trait could cause reduced level in the other trait.

Many approaches have been used by breeders to improve a population or select varieties for two or more traits economically important. Tandem selection involves selection for one trait until it reaches a satisfactory level, then you start to select for the other trait. If the two traits are positively correlated, then selection for the first trait leads to improvement in the second trait (Bernardo, 2010). Independent culling levels and index selection are two other strategies that are used to deal with selection for multiple traits (Godshalk et al., 1988). In independent culling, selection is done simultaneously but independently performed for all the traits and a specific level is considered for each trait. All the genotypes which do not reach this level are removed no matter how good the genotype is for other traits. Index selection involves selecting for several traits simultaneously on the basis of a single index value assigned to each candidate (Bos and Caligari, 2008). Both strategies are subjective because a certain weight is being given to each trait depending the researcher and the importance of the trait in the product profile (Yan and Frégeau-Reid, 2018).

The genotype by trait (GT) biplot has been utilized to study trait relationships and genotype evaluation in several crop species including soybean (Yan and Rajcan, 2002; Kocaturk et al., 2019), white lupin (Rubio et al., 2004), common bean (Hirpa et al., 2013; Gonzalez et al., 2006), cowpea (Oladejo et al., 2011), durum and bread wheat (Akcura, 2011; Reza et al., 2011).

A novel approach to genotype selection based on multiple traits, the genotype by yield*trait (GYT) biplot, was proposed by Yan and Frégeau-Reid (2018). In this approach, trait can be any breeding objective other than 
yield; it may be an agronomic trait, a grain quality, processing quality, or nutritional quality trait, or a disease resistance. The GYT biplot ranks genotypes based on their levels in combining yield with other target traits and at the same time shows their traits profile.

Sesame (Sesamum indicum L.) is a very ancient oilseed crop and one of the earliest domesticated oil crop in the world. It has acquired importance as a source of vegetable oil and proteins, good source of natural antioxidants (sesamin and sesamolin) which are unique for sesame and present in the oil (Ashri, 2007). Genetic and breeding improvement efforts in sesame have been limited and the results of such efforts slow to emerge. Ashri (2007) stated that the main reason for this limited success is that sesame is a crop mainly produced in developing countries and usually by smallholders. So far, none of the Consultative Group on International Agricultural Research (CGIAR) centers has been mandated to conduct research on sesame.

There is a growing demand for edible oil and biofuel and sesame seeds, which contain up to $50 \%$ oil (Arslan et al., 2007), could become an alternative source of income for small-scale farmers thus helping to alleviate rural poverty. Several researchers studied the association between yield component traits in sesame (Cagirgan et al., 2009; Boureima, 2012; Boureima et al., 2016). The results of these studies revealed that some of these traits are positively correlated with grain yield and others are unfavorably associated with yield. The objectives of this study were: (1) to study the relationship between different agronomic traits of sesame, (2) to select superior varieties based on multiple traits. The genotype profile of the selected varieties would be used to design a breeding program of sesame under rainfed conditions.

\section{MATERIALS AND METHODS}

\section{Germplasm and sites description}

Ten sesame genotypes were used in this study. They were SN-01-04; SN-01-06; SN 103; SN 203; SN 303; SN 403; HB 168; GK 01; DS 01; and 38-1-7. The trials were conducted during two main cropping seasons (2015 and 2016) at five locations in Niger: Maiguizawa $\left(13^{\circ} 58^{\prime} \mathrm{N}\right.$, $8^{\circ} 8^{\prime}$ E), Dadin Sarki $\left(13^{\circ} 44^{\prime} \mathrm{N}, 7^{\circ} 56^{\prime} \mathrm{E}\right)$, Gounaka $\left(13^{\circ} 40^{\prime} \mathrm{N}, 8^{\circ} 1^{\prime} \mathrm{E}\right)$, Hawandawaki $\left(13^{\circ} 21^{\prime} \mathrm{N}, 8^{\circ} 14^{\prime} \mathrm{E}\right)$ and Bandé $\left(13^{\circ} 10^{\prime} \mathrm{N}, 8^{\circ} 53^{\prime} \mathrm{E}\right)$. We used a Randomized Complete Block Design with five replications at all locations and years. The plot had 6 rows of $5 \mathrm{~m}$ length, 60 $\mathrm{cm}$ between rows and $20 \mathrm{~cm}$ between plants within the row. Sowing was done by hand drilling in rows and two weeks after sowing, plants were thinned to two per hole giving a total density of 166667 plants per hectare. Management practices such as insect, pest, disease and weed control were uniformly applied at all locations per years as per the recommendation for sesame growing. A composite N-P-K fertilizer (15-15-15) was applied at a rate of $80 \mathrm{~kg} \mathrm{ha}^{-1}$ before sowing and $50 \mathrm{~kg} \mathrm{ha}^{-1}$ of urea before flowering at each location.

In both seasons, data were recorded for plant height (HP), height to the first capsule on the main stem (HIPC), number of branches per plant (NR) and the number of capsules per plant (NCP). All these parameters were recorded from five randomly chosen plants in each plots. Flowering date (FL50) was determined when $50 \%$ of the plot population had at least one flower. Grain yield was evaluated after harvest from the four central rows.

\section{Statistical analysis}

A combined analysis of variance was performed from the mean data of the traits across all environments to detect the presence of Genotype by Environment Interaction (GEI). The adjusted mean values of the traits were used for the analysis of genotype by trait and trait associations. These mean values were standardized to mean zero and unity variance in order to minimize biases due to differences in scales of traits according to the formula suggested by Yan and Frégeau-Reid (2018):

$$
P_{i j}=\frac{T_{i j}-\bar{T}_{j}}{T_{j}}
$$

where $P_{i j}$ is the standardized value of genotype $i$ for trait or yield-trait combination $j$ in the standardized table, $T_{i j}$ is the original value of genotype $i$ for trait or yield-trait combination $j$ in the GT or GYT table, $T_{j}$ is the mean across genotypes for trait or yield-trait combination $j$, and $s j$ is the standard deviation for trait or yield-trait combination $j$.

The GT biplot (Figure 1) was constructed by plotting the PC1 scores against the PC2 scores for each genotype and each trait resulting from singular value decomposition (SVD) of the standardized GT data. SVD decomposes the GT table into genotype eigenvalues, trait eigenvalues, and singular values:

$$
P_{i j}=\left(d \lambda_{1}^{\alpha} S_{i 1}\right)\left(\lambda_{1}^{1-\alpha} \tau_{1 j} / d\right)+\left(d \lambda_{2}^{\alpha} S_{i 2}\right)\left(\lambda_{2}^{1-\alpha} \tau_{2 j} / d\right)+\varepsilon_{i j}
$$

Where $S_{i 1}$ and $S_{i 2}$ are the eigenvalues for PC1 and PC2, respectively, for genotype $i ; \tau_{1 j}$ and $\tau_{2 j}$ are the eigenvalues for $\mathrm{PC} 1$ and $\mathrm{PC} 2$, respectively for trait $j$; and $\varepsilon_{i j}$ is the residual from fitting the PC1 and PC2 for genotype $i$ on trait $j ; \lambda_{1}$ and $\lambda_{2}$ are the singular values for $\mathrm{PC} 1$ and PC2, respectively. $\alpha$ is the singular value partitioning factor.

The genotype by yield*trait (GYT) data (Table 1) was obtained as follows:

For the number of capsules per plant (NCP), the value for yield*trait combination was obtained by multiplying the yield value (RDT) with the trait value for each genotype (e.g., RDT*NCP). For the number of branches (NR), plant height (HP), the height to the first capsule on the main stem (HIPC) and flowering date (FL50), larger values are less favorable for higher seed yield in sesame crop. Thus, the values for the yield-trait combinations were obtained by dividing the yield value with the trait value for each genotype (e.g., RDT/NR) so that a larger value in the GYT table is always desirable.

The GT and GYT biplots were constructed using the GGEBiplotGUI package of $\mathrm{R}$ (3.5.2). 


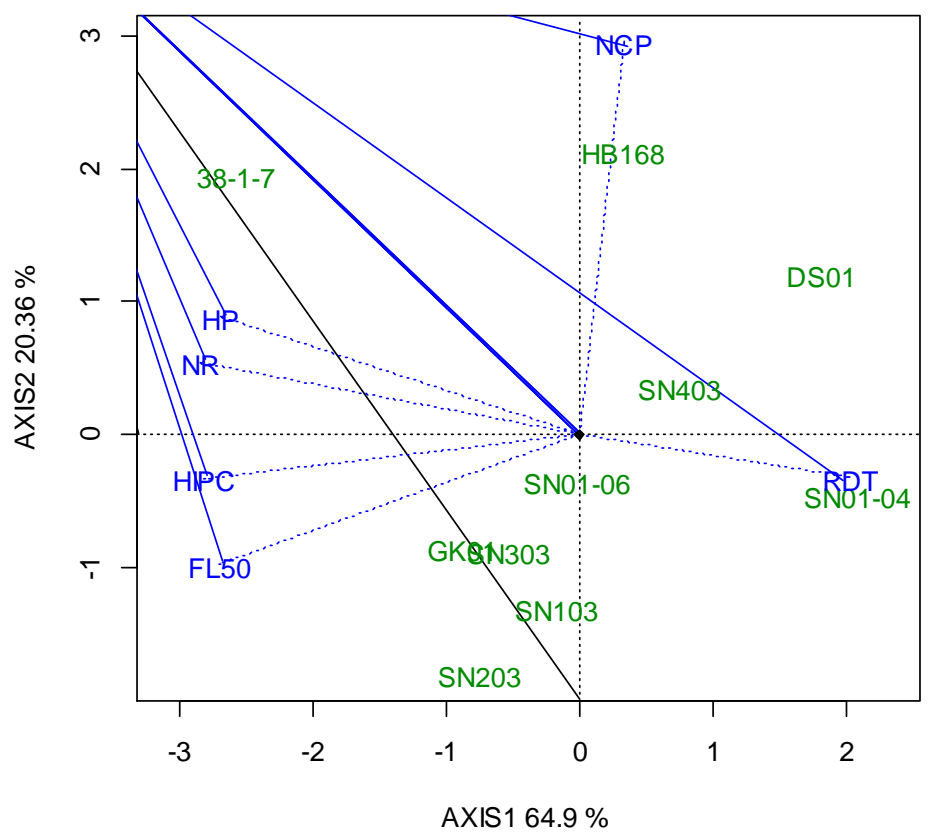

Figure 1. Genotype by trait biplot. The biplot was based on singular value decomposition of trait-standardized data ("Scaling = 1, Centering = 2") and trait-focused singular value partition ("SVP = 2"). The trait abbreviations' are: NR: number of branches; HP: height of the plant at maturity; HIPC: Height to the first capsule on the main stem; NCP: number of capsules per plant; FL50: flowering date; RDT: yield.

Table 1. Genotype by yield*trait combination data for 10 sesame genotypes.

\begin{tabular}{llllll}
\hline Genotype & rdt/NR & rdt/HP & rdt/HIPC & NCP*rdt & rdt/FL50 \\
\hline 38-1-7 & 113.12 & 5.01 & 8.74 & 38721.83 & 14.17 \\
SN403 & 270.54 & 8.49 & 16.82 & 54928.92 & 23.74 \\
DS01 & 428.65 & 8.47 & 18.49 & 56874.39 & 23.85 \\
GK01 & 197.05 & 7.11 & 13.26 & 44185.16 & 18.92 \\
HB168 & 187.11 & 6.90 & 15.78 & 49399.22 & 18.90 \\
SN01-04 & 335.14 & 7.81 & 17.66 & 42513.14 & 21.02 \\
SN01-06 & 220.54 & 7.28 & 14.21 & 45003.43 & 19.06 \\
SN103 & 207.22 & 7.29 & 13.45 & 41385.24 & 17.94 \\
SN203 & 195.75 & 6.71 & 12.27 & 37606.94 & 17.24 \\
SN303 & 202.97 & 6.87 & 12.89 & 41484.12 & 17.80 \\
Mean & $\mathbf{2 3 5 . 8 1}$ & $\mathbf{7 . 1 9}$ & $\mathbf{1 4 . 3 6}$ & $\mathbf{4 5 2 1 0 . 2 4}$ & $\mathbf{1 9 . 2 6}$ \\
\hline
\end{tabular}

The trait abbreviations' are: NR: number of branches; HP: height of the plant at maturity; HIPC: Height to the first capsule on the main stem; NCP: number of capsules per plant; FL50: flowering date; RDT: yield

\section{RESULTS AND DISCUSSION}

\section{Genotype by trait biplot (GT) and traits relationship}

The trait means for each of the 10 sesame varieties tested across 10 trials at five locations over 2 years are presented in Table 2 . The combined analysis of variance for all agronomical traits studied revealed that there were significant variation among the genotypes, environments (year, location, year $\mathrm{x}$ location) and genotype by environment interaction (Genotype x Location, Genotype $\mathrm{x}$ Year, and Genotype x Location x Year ) (Table 3). To visualize the relation between traits and traits profile of the genotypes, the genotype by trait (GT) data are displayed in Figure 1. The biplot was based on standardized data and explained $85.25 \%$ of the total variation. In the Figure 1, a vector is drawn from the biplot origin to each marker of traits to investigate the relationship between traits. Since the biplot explained a sufficient amount of the total variation, the Pearson correlation coefficient between two given traits is approximated by the cosine of the angle between their vectors (Yan and Rajcan, 2002). Thus, an angle smaller than $90^{\circ}$ indicates a positive correlation, an angle greater than $90^{\circ}$ indicates a negative correlation, and an angle of $90^{\circ}$ indicates that there is any correlation between the two traits. The interpretations of the biplot (Figure 1) is based on Yan and Frégeau-Reid (2018) who stated that the angle between a genotype and a trait indicates the relative level 
of the genotype for the trait. An acute angle indicates that the genotype is above-average for the trait; an obtuse angle indicates that the genotype is below-average for the trait; and a right angle indicates that the genotype is average for the trait. Also, the vector length of a trait indicates how well the trait is represented in the biplot; a relatively short vector indicates that the variation of the trait across genotypes is either small or not well represented in the biplot because of its weak or absence of correlation with other traits.

Based on these, observations made from Figure 1 evidenced that grain yield (RDT) is negatively correlated with plant height (HP), number of branches (NR), the height to the first capsule on the main stem (HIPC) and flowering date (FL50) both being closely correlated to each other. In our semi-arid conditions, taller sesame plants tend to have higher HIPC and more branches (NR) but with less fruiting zone.

The negative association between NR and RDT is explained by the fact that the increase in the number of branches may increase the intraplant competition (sourcesink) for resources to build grain yield.

Table 2. Genotype by trait for 10 sesame varieties for six traits. Each value is the mean across 10 trials from five location-years.

\begin{tabular}{cccccccc}
\hline Number & Genotype & NR & HP & HIPC & NCP & FL50 & RDT \\
\hline 1 & $38-1-7$ & 6.13 & 138.39 & 79.36 & 55.80 & 48.98 & 693.94 \\
2 & SN403 & 3.88 & 123.70 & 62.40 & 52.33 & 44.22 & 1049.68 \\
3 & DS01 & 2.36 & 119.57 & 54.80 & 56.13 & 42.48 & 1013.32 \\
4 & GK01 & 4.68 & 129.79 & 69.59 & 47.87 & 48.78 & 922.99 \\
5 & HB168 & 4.56 & 123.57 & 54.07 & 57.90 & 45.14 & 853.21 \\
6 & SN01-04 & 2.58 & 110.61 & 48.89 & 49.24 & 41.08 & 863.32 \\
7 & SN01-06 & 4.07 & 123.26 & 63.12 & 50.16 & 47.06 & 897.17 \\
8 & SN103 & 4.19 & 119.16 & 64.60 & 47.64 & 48.42 & 868.68 \\
9 & SN203 & 4.30 & 125.25 & 68.51 & 44.72 & 48.78 & 840.96 \\
10 & SN303 & 4.24 & 125.28 & 66.74 & 48.20 & 48.34 & 860.59 \\
& Mean & 4.10 & 123.86 & 63.21 & 51.00 & 46.33 & 886.39 \\
& LSD & 0.79 & 8.24 & 6.66 & 8.20 & 1.96 & 161.93 \\
\hline
\end{tabular}

The trait abbreviations' are: NR: number of branches; HP: height of the plant at maturity; HIPC: Height to the first capsule on the main stem; NCP: number of capsules per plant; FL50: flowering date; RDT: yield

Table 3. Mean squares for different agronomical traits recorded on sesame genotypes across locations and years

\begin{tabular}{llllllll}
\hline Source of variation & DF & HIPC $(\mathbf{c m})$ & HP(cm) & NR & NCP & FL50 & RDT $\left(\mathbf{k g ~ h a} \mathbf{~}^{-1}\right)$ \\
\hline Rep & 4 & 11.9 & 343.2 & 1.7 & 209.9 & 9.6 & 74313 \\
Gen & 9 & $3912.5^{* *}$ & $2593.8^{* *}$ & $55.7^{* *}$ & $1025.6^{* *}$ & $423.5^{* *}$ & $460923^{* *}$ \\
Loc & 4 & $1015.6^{* *}$ & $4574.1^{* *}$ & $82.9^{* *}$ & $4335.3^{* *}$ & $1628.6^{* *}$ & $460923^{* *}$ \\
Year & 1 & $866.7^{* *}$ & $25634.1^{* *}$ & $96.0^{* *}$ & $48282.3^{* *}$ & $139.4^{* *}$ & $16797738^{* *}$ \\
Gen*loc & 36 & 92.6 & 241.5 & $4.4^{* *}$ & $415.1^{* *}$ & $5.5^{*}$ & 82203 \\
Gen*Year & 9 & $530.6^{* *}$ & $928.7^{* *}$ & $17.6^{* *}$ & $951.9^{* *}$ & 19.5 & $176074 * *$ \\
Loc*Year & 4 & $18345.7^{* *}$ & $7233.5^{* *}$ & $10.7^{* *}$ & $5130.6^{* *}$ & $889.1^{* *}$ & $2573782^{* *}$ \\
Gen*Loc*Year & 36 & 143.8 & 214.9 & $2.8^{* *}$ & 202.4 & $5.7^{* *}$ & $84015^{*}$ \\
Residual & 368 & 122.2 & 294.3 & 2.8 & 236.3 & 3.3 & 58462 \\
\hline
\end{tabular}

The trait abbreviations' are: NR: number of branches; HP: height of the plant at maturity; HIPC: Height to the first capsule on the main stem; NCP: number of capsules per plant; FL50: flowering date; RDT: yield; Rep: repetition; Gen: genotype; Loc: location

The near right angle between yield and the number of capsules per plant evidenced that there is no correlation between these two traits. These statements can be verified from Table 4 and are in agreement with Van Rheenen (1981) who found no relationship between number of capsules and seed yield under rainfed conditions in Nigeria and in a world survey. These results are opposed to other studies (Boureima et al., 2016; Zhou et al., 2018) who reported a positive association between NCP and seed yield. This is why Cagirgan et al. (2009) applied selection for number of capsules in the pairwise $F_{2}$ populations of determinate and indeterminate crosses of sesame and estimated genetic gains comparatively by $\mathrm{F}_{3}$ generation means.
Table 4. Pearson correlations between traits across 10 genotypes.

\begin{tabular}{llllll}
\hline & FL50 & HIPC & HP & NCP & NR \\
\hline HIPC & $0.87^{* *}$ & & & & \\
HP & $0.72^{*}$ & $0.88^{* *}$ & & & \\
NCP & -0.39 & -0.21 & 0.19 & & \\
NR & $0.80^{* *}$ & $0.84^{* *}$ & $0.88^{* *}$ & 0.06 & \\
RDT & -0.50 & -0.49 & -0.45 & 0.01 & -
\end{tabular}

$0.67 *$

The trait abbreviations' are: NR: number of branches; HP: height of the plant at maturity; HIPC: Height to the first capsule on the main stem; NCP: number of capsules per plant; FL50: flowering date; RDT: yield 
The GT biplot (Figure 1) also shows the trait profiles of the genotypes. For example genotype 38-1-7 had the highest HP, HIPC, NR, late flowering and was the worst in terms of grain yield. Genotype HB168 had more capsules per plant but was not the high yielding genotype. This behavior is illustrated by the near right angle between NCP and RDT. Despite the fact that GT biplot approach is a powerful tool in evidencing the relationship between traits and trait profiles of the genotypes, it is not helpful in making decision on which genotype to select or to discard (Yan and Frégeau-Reid, 2018). The yield-trait combination displayed in Figure 2 showed that these combination traits tend to be positively correlated with each other since they have all yield as a component. Figure 2 was based on values from Table 1 where highest values were suitable for each combination of trait with yield.

\section{Trait profile of the genotypes}

Figure 3 is the polygon view of yield-traits combinations and genotypes. The polygon was constructed by connecting the markers of the genotypes that are farthest away from the biplot origin so that all other genotypes are contained in the polygon. For each polygon side, a line was drawn to start from the biplot origin and to be perpendicular to the polygon side. These lines divided the yield-trait combinations into 4 sectors out of which 2 sectors are without traits. Sectors without traits indicate that the genotypes falling in these sectors including the vertex genotypes, for instance genotypes 1 , $7,8,9$ and 10 had low values for all the traits studied. The genotype placed on a vertex is the most performer for the yield-trait combinations placed within the corresponding sector (Yan and Tinker, 2003; Yan et al., 2007; Yan and Frégeau-Reid, 2018). Thus, the genotype 3 (DS01) had the largest values for $\mathrm{rdt} / \mathrm{FL} 50, \mathrm{rdt} / \mathrm{HP}, \mathrm{rdt} / \mathrm{HIPC}$ and $\mathrm{rdt} / \mathrm{NR}$ meaning that DS01 was the best genotype combining seed yield with earliness in flowering, medium height, short height from the ground to the first capsule on the main stem and less branches. Genotype 2 (SN403) was the best in combining seed yield with the number of capsules. Early flowering and medium height genotypes are very useful for semi-arid environments where terminal drought and wind are very common during the cropping season. This statement is supported by the behavior of a mutantvariety "Birkan" originated from Mediterranean region and introduced in Africa. This mutant-variety "Birkan" performs well in short-duration of rainfall conditions by flowering early due to its photoperiod insensitivity, has short stature and low length to the first capsule. Boureima et al. (2009) and Diouf et al. (2010) attempted to induce genetic variability for these traits in some African sesame cultivars by using gamma rays because criteria for superior genotype selection must be based on multiple traits that are considered as breeding targets (Yan, 2014).

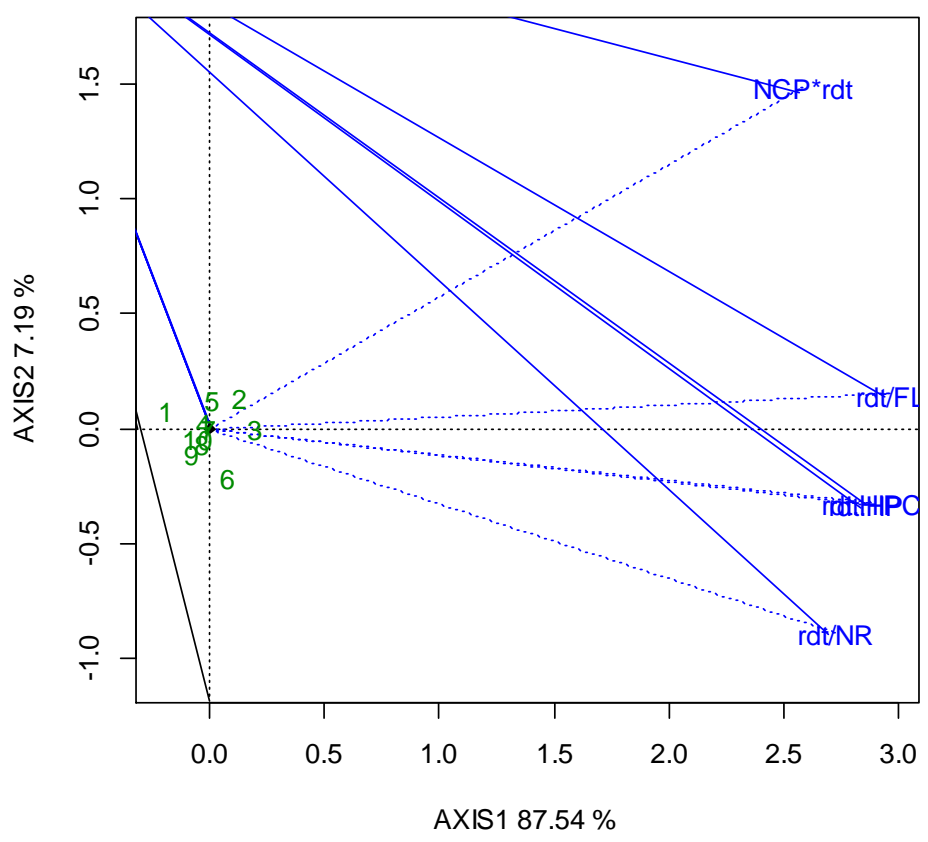

Figure 2. The Tester Vector view of the genotype by yield*trait (GYT) biplot to show associations among the yield-trait combinations. The biplot was based on singular value decomposition of the standardized GYT table ("Scaling = 1, Centering = 2"). The trait-focused singular value partition ("SVP =2") was used. The trait abbreviations' are: NR: number of branches; HP: height of the plant at maturity; HIPC: Height to the first capsule on the main stem; NCP: number of capsules per plant; FL50: flowering date; RDT: yield 


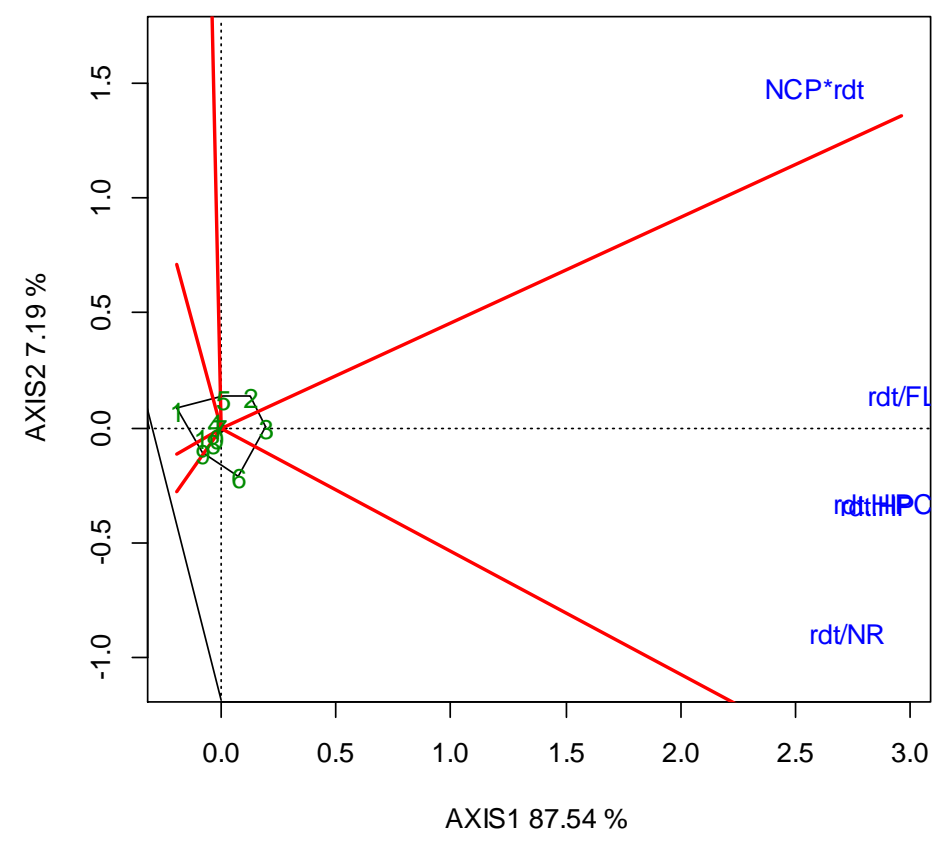

Figure 3. The which-won-where view of the genotype by yield*trait (GYT) biplot to highlight genotypes with outstanding profiles. The biplot was based on singular value decomposition of the standardized GYT table ("Scaling = 1, Centering = 2"). The traitfocused singular value partition ("SVP $=2$ ") was used. Numbers referred to genotype numbers. The trait abbreviations' are: NR: number of branches; HP: height of the plant at maturity; HIPC: Height to the first capsule on the main stem; NCP: number of capsules per plant; FL50: flowering date; RDT: yield

\section{Rank of the genotypes based on their yield-trait combinations}

The Figure 4 is the average tester coordination (ATC) view to focus on comparison among genotypes. The small circle in the biplot represents the placement of the average yield-trait combination. The horizontal line passing through the biplot origin is called the average tester axis (ATA). The ATA points from left to wright, toward higher means of yield-trait combination and serves to rank the genotypes based on their overall performance (Yan and Frégeau-Reid, 2018). DS01 (genotype 3) had the highest mean, followed by SN403 (genotype 2) and SN-01-04 (genotype 6) whereas 38-1-7 (genotype 1) had the lowest mean of yield-trait combination.

The vertical line, perpendicular to the ATA separates genotypes in two groups. All the genotypes at the right part of this vertical line perform better than average and all those at the left side performed below average. The vertical line serves also to show whether a genotype had a complete or balanced trait profile. Genotypes which are close to ATA tend to have balanced trait profiles whereas those placed far away from the ATA in both directions tend to have evident strengths and/or weaknesses (Frutos et al., 2014). Based on these principles, the best ranked genotypes based on yield-trait combinations are DS01 (genotype 3) followed by SN403 (genotype 2). The yieldtrait combination biplot is a powerful tool to assess which accessions were good for which trait (s) that would help as excellent baseline information for sesame improvement. The superiority of a genotype should not only be measured by its levels in individual traits but more importantly by its levels in combining yield with other target traits (Yan and Frégeau-Reid, 2018). For example, in semi-arid regions where the rainfed season is very short (3 months), a high yielding sesame cultivar may not be adopted by farmers if it is late in flowering or very tall. Taller sesame varieties are generally susceptible to lodging during wind events specially if the number of capsules is high. 


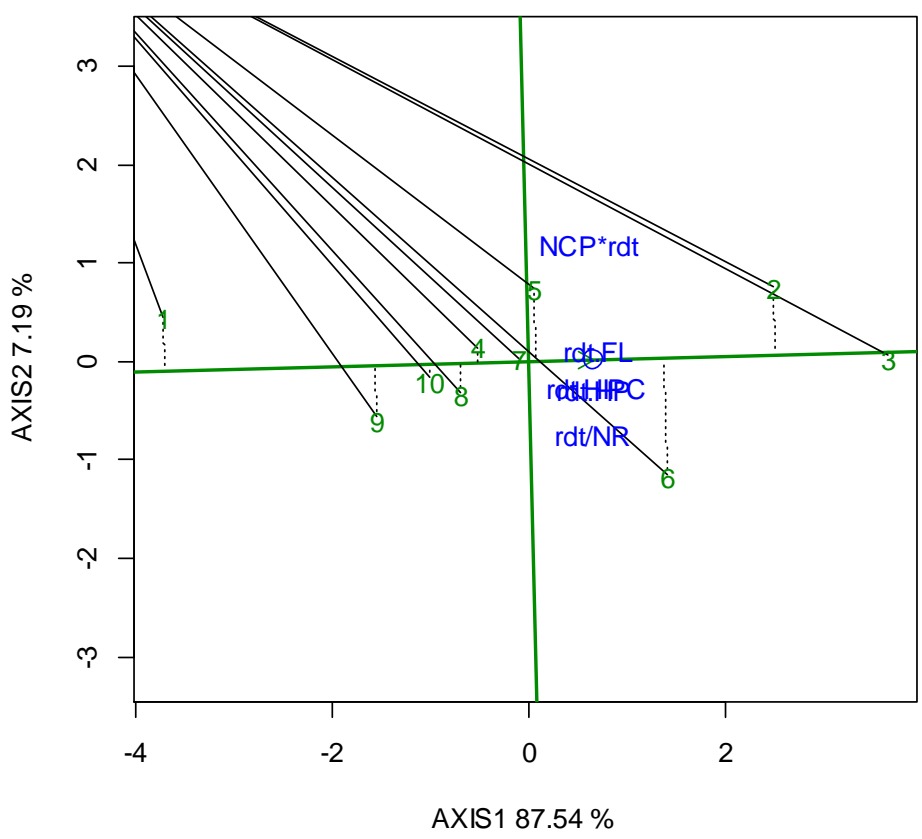

Figure 4. The Average Tester Coordination view of the genotype by yield*trait (GYT) biplot to rank the genotypes. Numbers referred to genotype numbers. The trait abbreviations' are: NR: number of branches; HP: height of the plant at maturity; HIPC: Height to the first capsule on the main stem; NCP: number of capsules per plant; FL50: flowering date; RDT: yield

\section{ACKNOWLEDGEMENTS}

The authors are grateful to the West Africa Agricultural Productivity Program (WAAPP-Niger) for funding the research project $\mathrm{N}^{\circ} 005 / 2015 / \mathrm{UCP} / \mathrm{PPAAO}$.

\section{LITERATURE CITED}

Akcura, M. 2011. The relationships of some traits in Turkish winter bread wheat landraces. Turk J Agric For. 35:115-125.

Arslan, C., B. Uzun, S. Ulger and M.I. Cagirgan. 2007.

Determination of oil content and fatty acid composition of sesame mutants suited for intensive management conditions. Journal of the American Oil Chemists Society 84:917-920.

Ashri, A. 2007. Sesame (Sesamum indicum L.). In: Singh RJ (ed) Genetics Resources, Chromosome Engineering, and Crop Improvement, Vol.4, Oilseed Crops, CRC Press, Boca Raton, FL, USA. pp. 231-289.

Bernardo, R. 2010. Breeding for quantitative traits in plants. Second edition, Stemma press, Minnesota, 390 p.

Boss, I. and P. Caligari. 2008. Selection methods in plant breeding. 2.ed. Springer, Dordrecht, Netherlands.

Boureima, S., M. Diouf, A.I. Amoukou and P. Van Damme. 2016. Screening for sources of tolerance to drought in sesame induced mutants: Assessment of indirect selection criteria for seed yield. Int. J. Pure App. Biosci. 4 (1): 45-60.

Boureima, S. 2012. Amélioration variétale du sésame (Sesamum indicum L.) par mutation induite: effet de la mutagenèse sur la tolérance à la sécheresse et la productivité. $\mathrm{PhD}$ thesis, ISBN 978-90-5989-506-5, Ghent University Press, Ghent, Belgium 194 pp.

Boureima, S., M. Diouf, R.S. Silme, T. Diop, P. Van Damme and M.I. Cagirgan. 2009 Radiosensitivity of african sesame cultivars to gamma-rays. Turkish Journal of Field Crops 14:181-190.
Cagirgan, M.I., S. Ozerden and M. O. Ozbas. 2009. Agronomic trait assessment and selection for number of capsules in determinate $\mathrm{x}$ indeterminate crosses of sesame. Turkish Journal of Agriculture and Forestry 33:231-241.

Diouf, M., S. Boureima, T. Diop and M.I. Cagirgan. 2010. Gamma rays-induced mutant spectrum and frequency in sesame. Turkish Journal of Field Crops 15:99-105.

Frutos, E., M.P. Galindo and V. Leiva. 2013. An interactive biplot implementation in $\mathrm{R}$ for modeling genotype-byenvironment interaction. Stoch Environ Res. Risk Assess. 28 (7):1629-1641.

Godshalk, E.B., D.H. Timothy and J.C. Burns. 1988. Effectiveness of index selection for switchgrass forage yield and quality. Crop Sci. 28:825-830.

González, A.M., A.B. Monteagudo, P.A. Casquero, A.M. De Ron and M. Santalla. 2006. Genetic variation and environmental effects on agronomical and commercial quality traits in the main European market classes of dry bean. Field Crops Res. 95:336-347.

Hirpa, L., D. Nigussie, G. Setegn, B. Geremew and M. Firew. 2013. Multivariate analysis as a tool for indirect selection of common bean genotypes (Phaseolus vulgaris L) for soil acidity tolerance under field conditions. Sci Technol. Arts Res J. 2: 07-15.

Kocaturk, M., P. Cubukcu, A. T. Goksoy, M. Sincık, E. Ilker, A. Kadıroglu, Y. Vurarak, Y. Sahın, M. Karakus and U. A. Yildırım. 2019. GGE Biplot Analysıs Of Genotype × Environment Interaction In Soybean Grown as a Second Crop. Turkish Journal of Field Crops 24 (2): 145-154.

Oladejo, A.S., R.O. Inwale and I.O. Obisesan. 2011. Interrelationships between grain yield and other physiological traits of cowpea cultivars. Afr Crop Sci J. 19: $189-200$. 
Reza, M. and A. Ahmed. 2011. Graphic analysis of trait relations and genotype evaluation in durum wheat. J. Crop. Improv. 25: 680-696.

Rubio, J., J.I. Cubero, L.M. Martin, M.J. Suso and F. Flores. 2004. Biplot analysis of trait relations of white lupin in Spain. Euphytica 135: 217-224.

Van Rheenen, H. A. 1981. The desirability of three versus one flowers and capsules per leaf axil in sesame (Sesamum indicum L.). In : Sesame : Status and improvement. Proceeding of expert consultation, FAO, 1981, Rome (Dec. 1980), pp 8-12.

Yan, W., N.A. Tinker, S. Molnar, J. Fregeau-Reid and A. McElroy. 2007. Associations among oat traits and their responses to the environment in North America. J. Crop Improv. 20:1-29.
Yan, W. 2014. Genotype-by-trait data analysis and decisionmaking. Chapter 9. In: Crop variety trials: data management and analysis, ed. Yan, W., 163-186, John Wiley \& Sons, INC., New York.

Yan, W. and J. Frégeau-Reid. 2018. Genotype by Yield*Trait (GYT) Biplot: a Novel Approach for Genotype Selection based on Multiple Traits. Scientific Reports 8:8242.

Yan, W. and I.R. Rajcan. 2002. Biplot analysis of test sites and trait relations of soybean in Ontario. Can. J. Plant Sci. 42:11-20.

Zhou, R., K. Dossa, D. Li, J. Yu, J. You, X. Wei and X. Zhang. 2018. Genome-Wide Association Studies of 39 Seed YieldRelated Traits in Sesame (Sesamum indicum L.). Int. J. Mol. Sci. 19: 2794. 